\title{
Exotic Mesons at JLab Before 2013? The Search for New Forms of Matter at CLAS
}

\author{
Craig Bookwalter \\ on behalf of the HyCLAS collaboration \\ Department of Physics \\ Florida State University \\ Tallahassee, FL 32306
}

\begin{abstract}
A proposal to search for exotic mesons in photoproduction has been accepted for running at Thomas Jefferson National Accelerator Facility, using the CEBAF Large Acceptance Spectrometer in Hall B. This program will bolster previously-thin statistics in many photoproduction channels, primarily those with charged particles in the final state, as well as seeking to confirm earlier findings in neutral channels, if possible. The promise of the neutral $3 \pi$ channel is discussed. In addition, the experiment seeks to study the spectrum of both exotic and ordinary strangeonia. Limitations of the CLAS detector for meson spectroscopy are discussed, as well as possible solutions to minimize such limitations.
\end{abstract}

Keywords: exotic, hybrid, gluonic, meson, spectroscopy, photoproduction

PACS: $12.39 . \mathrm{Mk}$

\section{INTRODUCTION}

Exotic mesons are most generally defined as particles with zero baryon number and $J^{P C}$ values not allowed by the quark model. Physically, possibilities include a pair of quarks with glue as an addition constituent (known as a gluonic hybrid), a state consisting only of glue (a glueball), or combinations of mesons or baryons into four- or six-quark composites.

Mesons with exotic $J^{P C}$ were first predicted in 1968 by Jon Rosner from Tel Aviv University using Regge phenomenology [1], but foundations of the field were laid with the exotic meson mass predictions of the flux-tube model, developed in 1983 by Nathan Isgur and Jack Paton [2]. Twelve years later, Frank Close and Ted Barnes extended the work of Isgur and Paton to predict the masses of flux-tube excitations with non-exotic $J^{P C}[3]$.

Experimentally, the first hints of exotics were observed in pion production at CERN during the GAMS experiment. The GAMS collaboration reported a small enhancement at $1400 \mathrm{MeV}$ with $J^{P C}=1^{-+}$[4]. Later collaborations at VES in Russia and KEK in Japan observed similar behavior in pion production at $1400 \mathrm{MeV}$ in the $1^{-+}$wave, but their phase analyses differed significantly [5], [6]. None of these collaborations were willing to label their observation at $1400 \mathrm{MeV}$ as an exotic meson.

In 1997 and 1998, the E852 collaboration from Brookhaven National Laboratory published results claiming the existence of $1^{-+}$exotics at both $1400 \mathrm{MeV}$ and 1600 $\mathrm{MeV}$ in pion production $[7,8]$. Their claim on the $\pi_{1}(1400)$ was backed by evidence

CP947, VII Latin American Symposium on Nuclear Physics and Applications

edited by R. Alarcon, P. L. Cole, C. Djalali, and F. Umeres

(C) 2007 American Institute of Physics 978-0-7354-0461-8/07/\$23.00 
TABLE 1. A sample of measured photoproduction cross sections. Note the small yields in all channels.

\begin{tabular}{|ccc|}
\hline Reaction & $E_{\gamma}[\mathrm{GeV}]$ & Events \\
\hline$\gamma p \rightarrow p \pi^{+} \pi^{-}$ & 9.3 & 3500 \\
$\gamma p \rightarrow p \pi^{+} \pi^{-}$ & 19.3 & 20980 \\
\hline$\gamma p \rightarrow p \pi^{+} \pi^{-} \pi^{\circ}$ & 4.7 & 1606 \\
$\gamma p \rightarrow p \pi^{+} \pi^{-} \pi^{\circ}$ & 9.3 & 1195 \\
$\gamma p \rightarrow p \pi^{+} \pi^{-} \pi^{\circ}$ & $4.7-5.8$ & 3001 \\
$\gamma p \rightarrow p \pi^{+} \pi^{-} \pi^{\circ}$ & $6.8-8.2$ & 7297 \\
$\gamma p \rightarrow p \pi^{+} \pi^{-} \pi^{\circ}$ & $20-70$ & 14236 \\
$\gamma p \rightarrow n \pi^{+} \pi^{+} \pi^{-}$ & $4.7-5.8$ & 1723 \\
$\gamma p \rightarrow n \pi^{+} \pi^{+} \pi^{-}$ & $6.8-8.2$ & 4401 \\
$\gamma p \rightarrow n \pi^{+} \pi^{+} \pi^{-}$ & $16.5-20$ & 3781 \\
\hline$\gamma p \rightarrow p \pi^{+} \pi^{-} \pi^{+} \pi^{-}$ & $4.0-6.0$ & $\sim 300$ \\
$\gamma p \rightarrow p \pi^{+} \pi^{-} \pi^{+} \pi^{-}$ & $6.0-8.0$ & $\sim 500$ \\
$\gamma p \rightarrow p \pi^{+} \pi^{-} \pi^{+} \pi^{-}$ & $8.0-12.0$ & $\sim 500$ \\
$\gamma p \rightarrow p \pi^{+} \pi^{-} \pi^{+} \pi^{-}$ & $12.0-18.0$ & $\sim 400$ \\
$\gamma p \rightarrow p \pi^{+} \pi^{-} \pi^{+} \pi^{-}$ & $15.0-20.0$ & $\sim 400$ \\
$\gamma p \rightarrow p \pi^{+} \pi^{-} \pi^{\circ} \pi^{\circ}$ & $20-70$ & 8100 \\
\hline$\gamma p \rightarrow p \pi^{+} \pi^{-} \pi^{+} \pi^{-} \pi^{\circ} \pi^{\circ}$ & 19.5 & 2553 \\
\hline$\gamma p \rightarrow \Delta^{++} \pi^{+} \pi^{-} \pi^{-}$ & $4.0-6.0$ & $\sim 200$ \\
$\gamma p \rightarrow \Delta^{++} \pi^{+} \pi^{-} \pi^{-}$ & $6.0-8.0$ & $\sim 200$ \\
$\gamma p \rightarrow \Delta^{++} \pi^{+} \pi^{-} \pi^{-}$ & $8.0-12.0$ & $\sim 200$ \\
\hline$\gamma p \rightarrow \Delta^{++} \pi^{+} \pi^{-} \pi^{-}$ & $12.0-18.0$ & $\sim 200$ \\
\hline
\end{tabular}

from the Crystal Barrel collaboration in 1998 [9]. Both the $\pi_{1}(1400)$ and the $\pi_{1}(1600)$ claims were met with considerable resistance. At present, the community generally acknowledges the existence of the $\pi_{1}(1600)$, but the $\pi_{1}(1400)$ remains debatable.

During the running of E852, the community built a proposal for an experiment to definitively observe and study gluonic matter. Timely theoretical work by Frank Close and Phillip Page showed that in photoproduction, exotics were likely to be produced at the same rates as ordinary matter, as opposed to a factor of five reduction in exotics versus ordinary matter for pion production [10]. In addition, current world photoproduction data is thin at best in many relevant reaction channels (shown in Table 1) [11]. This lack of data illustrates the need for a high-statistics, dedicated experiment for the study of exotic mesons, and the GlueX experiment has been designed to fill that role.

However, GlueX requires an upgrade to the Jefferson Lab accelerator, as well as the construction of a new experimental hall. While the upgrade is one of the Department of Energy's top scientific priorities, commissioning is not likely to take place until 2013.

With this in mind, a collaboration of physicists submitted a proposal for the HyCLAS experiment in January 2004, with the idea to search for exotics prior to the commissioning of GlueX using the CLAS detector in Hall B. The Jefferson Lab Program Advisory Committee (PAC) approved the proposal with an "A" rating for 35 days of beam time. 


\section{DETAILS ON THE HYCLAS PROPOSAL}

The HyCLAS experiment seeks to use the Jefferson Lab accelerator at its current maximum beam energy of $6 \mathrm{GeV}$, with tagged photons emerging in the range of 5 to $6 \mathrm{GeV}$. A demonstration of feasibility for the purpose of the proposal was prepared by performing a partial-wave analysis of 8 days of photoproduction data from the $\mathrm{g} 6 \mathrm{c}$ run, where results showed tantilizing evidence of exotics [11]. The full run is expected to produce statistics on par with those of E852, numbering in the millions of events per channel.

The available channels for analysis from the HyCLAS data include all the chargedpion final states, and possibly those including multiple $\pi^{0}$ or $\eta$, depending on detector configuration. Among the neutral channels, the $\eta \pi$ and $\eta^{\prime} \pi$ systems would be especially interesting due to their study in E852, while the neutral $3 \pi$ channel $\left(\gamma p \rightarrow p \pi^{+} \pi^{-} \pi^{0}\right)$ could provide a unique and rewarding challenge. This is due to the observation of the $\pi_{1}(1600)$ in four E852 reaction channels none with charge exchange, just like the neutral $3 \pi$ [12], [13], [14], [15]. The lack of charge exchange implies the exchange of a pomeron, and pomerons could be a source of glue-rich decays. However, the analysis of the neutral $3 \pi$ channel could be challenging, depending on the detector setup. If CLAS is equipped with some sort of small-angle neutral reconstruction, then the $\pi^{0}$ could be detected directly, and with a proton reconstructed through missing mass, cuts could be made to ensure a smaller momentum transfer, thus cleaning the data set of excited baryons. However, without such neutral reconstruction at small angles, the proton must be detected directly by CLAS and the $\pi^{0}$ reconstructed via missing mass. This inherently involves a large momentum transfer to the proton, and half-spin waves will be required for a complete partial wave analysis.

In addition, HyCLAS provides an opportunity to study the spectrum of $s \bar{s}$ mesons. Of twenty-two predicted states, only five have been observed, and photoproduction ought to be a rich hunting ground for strangeonia, both ordinary and exotic. The study of strangeonia is important, because the strange and charm quarks are of intermediate mass. Theoretical calculations are best performed with heavy quark masses, but most matter in the universe is made with light quarks, so understanding this interface can help the community understand the relationship between the light and heavy quarks.

\section{THE CEBAF LARGE ACCEPTANCE SPECTROMETER}

CLAS in Hall B is designed for baryon spectroscopy. It is divided axially into six sections (like an orange) with the beam flowing through the axis of division. Each section has consecutive layers of detection equipment, such as drift chambers for chargedparticle tracking, time-of-flight counters and Cerenkov detectors for particle identification, and lead-scintillator sampling calorimeters for energy measurement. The six sections combine to form a solid-angle acceptance of nearly- $4 \pi$ steradians; however, CLAS performs badly in the forward angles, where most exotic-rich events are likely to fall.

Several changes have been proposed to minimize the weaknesses of CLAS in the forward direction: movement of the target as far upstream as allowable to minimize the angluar size of the hole, and the reduction of the strength of the torus magnet by half minimizing the loss of negatively-charged particles through the forward hole. In addi- 
tion, discussion continues about the inclusion of a new calorimeter (the inner calorimeter) designed by the Deeply-Virtual Compton Scattering collaboration at Jefferson Lab for reconstructing $\pi^{0}$ decays. The inner calorimeter was designed for "plugging" the forward hole in the acceptance of CLAS, and preliminary physics studies show promising results [16], but engineering concerns may scuttle the plans for its inclusion. In either case, a new hodoscope has been proposed to assist in tracking charged particles at small angles, either to veto them upon entry to the inner calorimeter or to attempt to catch them escaping through the forward hole.

\section{CONCLUSION}

The HyCLAS collaboration expects the experiment run by the beginning of 2008. While GlueX will still be the definitive exotic meson spectroscopy experiment when it runs, researchers have the opportunity to confirm earlier findings as well as direct the attention of the future GlueX program with HyCLAS.

\section{ACKNOWLEDGMENTS}

I would like to thank the HyCLAS collaborators for their wisdom and the organizers of the Symposium for their hospitality.

\section{REFERENCES}

1. J. L. Rosner, Phys. Rev. Lett. 21, 950 (1968).

2. N. Isgur and J. E. Paton, Phys. Lett. B 124, 247 (1983).

3. T. Barnes, F. E. Close and E. S. Swanson, Phys. Rev. D 52, 5242 (1995)

4. D. Alde et al. [IHEP-Brussels-Los Alamos-Annecy(LAPP) Collaboration], Phys. Lett. B 205, 397 (1988).

5. G. M. Beladidze et al. [VES Collaboration], Phys. Lett. B 313, 276 (1993).

6. H. Aoyagi et al., Phys. Lett. B 314, 246 (1993).

7. D. R. Thompson et al. [E852 Collaboration], Phys. Rev. Lett. 79, 1630 (1997)

8. G. S. Adams et al. [E852 Collaboration], Phys. Rev. Lett. 81, 5760 (1998).

9. W. Dunnweber, K. Huttmann, W. Roethel (1997), given at the 7th International Conference on Hadron Spectroscopy (Hadron 97), Upton, NY, 25-30 Aug 1997.

10. F. E. Close and P. R. Page, Phys. Rev. D 52, 1706 (1995)

11. P. Eugenio, C. Salgado, and D. Weygand, (2003), propsal submitted to the Jefferson Lab PAC 25

12. J. Kuhn et al. [E852 Collaboration], Phys. Lett. B 595, 109 (2004)

13. M. Lu et al., Phys. Rev. D 65, 032002 (2005)

14. S. U. Chung et al., Phys. Rev. D 65, 072001 (2002).

15. D. L. Stienike (1998), PhD thesis, University of Notre Dame

16. C. Bookwalter, P. Eugenio, and A. Ostrovidov, DOE Journal of Undergraduate Research VII (2006). 